\title{
MoCa: Una aplicación móvil para registrar síntomas de pacientes con cáncer
}

\author{
Beatriz A. González-Beltrán ${ }^{1}$, Lizbeth Gallardo-López ${ }^{1}$, \\ Roberto García-Madrid ${ }^{2}$, Marco V. Ferruzca-Navarro² ${ }^{2}$ Alejandro Reyes-Ortíz ${ }^{1}$ \\ 1 Universidad Autónoma Metropolitana, campus Azcapotzalco, División de Ciencias \\ Básicas e Ingeniería, Ciudad de México, México \\ 2 Universidad Autónoma Metropolitana, campus Azcapotzalco, División de Ciencias \\ y Artes para el Diseño, Ciudad de México, México \\ \{bgonzalez, glizbeth, gmra, mvfn, jaro\}@azc.uam.mx
}

\begin{abstract}
Resumen. Las últimas estadísticas de la Agencia Internacional para la Investigación del Cáncer, la agencia especializada en cáncer de la Organización Mundial de la Salud muestra un incremento en la incidencia de cáncer en el mundo. En 2012, los casos de cáncer aumentaron a un estimado de 14 millones por año. Esta enfermedad aumentará a 22 millones de casos nuevos por año para 2030. Por lo tanto, se necesitan nuevos sistemas de software para el cuidado de la salud para ayudar a la detección temprana, el diagnóstico, el tratamiento, la supervivencia y la atención al final de la vida de los pacientes con cáncer, sus familias y los médicos. Los pacientes con cáncer son atendidos por sus médicos en sus tratamientos contra el cáncer y en los controles de rutina. Los pacientes deben estar conscientes de su estado de salud para proporcionar información a sus médicos. De esta manera, los pacientes mantienen una mejor calidad de vida y los médicos toman mejores decisiones clínicas. Registrar los síntomas de un paciente es vital, especialmente durante los tratamientos contra el cáncer. En este artículo se presenta MoCa, una aplicación móvil para el registro de síntomas de pacientes con cáncer. Se realizaron entrevistas con los usuarios. MoCa permite a los pacientes registrar sus síntomas usando una figura del cuerpo o el teclado. Se realizaron pruebas cualitativas a pacientes. Se encontró que los pacientes respondieron positivamente, tienen confianza al registrar sus síntomas y a comunicarse con sus médicos de manera precisa.
\end{abstract}

Palabras clave: aplicación móvil, salud móvil, síntoma, cáncer, ssabilidad.

\section{MoCa: A Mobile Application for Registering Symptoms of Cancer Patients}

\begin{abstract}
Latest statistics of the International Agency for Research on Cancer, the specialized cancer agency of the World Health Organization shows an increasing global incidence of cancer. In 2012, cancer cases rose to an estimated of 14 million per year. The burden of cancer will
\end{abstract}


Beatriz A. González-Beltrán, Lizbeth Gallardo-López, Roberto García-Madrid, et al.

increase to 22 million new cases per year by 2030. Therefore, new healthcare software systems are needed to help the early detection, diagnosis, treatment, survivorship and end-of-life care for cancer patients, their families, and clinicians. Cancer patients are followed by their clinicians in their cancer treatments and in routine checks. Patients must be aware of their health status to give information to their clinicians. In this manner, patients maintain their best quality of life and clinicians take better clinical decisions. Recording their symptoms is vital, particularly during cancer treatments. We present MoCa, a mobile application for registering symptoms of cancer patients. We conduct interviews with users. MoCa allows patients to register their symptoms using a body figure or the keyboard. We performed a qualitative test to patients. We find that patients responded positively, they are confident with registering their symptoms and to communicate with theirs clinicians in a precise way.

Keywords: mobile application, mobile health, symptom, cancer, usability.

\section{Introducción}

Las últimas estadísticas de la Agencia Internacional para la Investigación del Cáncer muestran un incremento en la incidencia de cáncer en el mundo. En particular, los sistemas de cuidado de la salud esperan un aumento de enfermedades crónicas como enfermedades cardiovasculares, diabetes, enfermedades respiratorias crónicas y cáncer [12. En 2012, los casos de cáncer aumentaron a un estimado de 14 millones por año. Esta enfermedad aumentará a 22 millones de casos nuevos por año para 2030. Por lo tanto, se necesitan nuevos sistemas de software para el cuidado de la salud para ayudar a la detección temprana, el diagnóstico, el tratamiento, la supervivencia y la atención al final de la vida de los pacientes con cáncer, sus familias y los médicos.

Los pacientes diagnosticados con cáncer requieren un régimen específico de tratamiento que abarque una o más modalidades, como la cirugía y/o la radioterapia, y/o la quimioterapia 12. Los pacientes con cáncer son atendidos por sus médicos en sus tratamientos contra el cáncer y en los controles de rutina. Los médicos alientan a los pacientes a registrar sus síntomas. Registrar los síntomas de un paciente es vital, particularmente durante los tratamientos contra el cáncer porque cada persona reacciona de diferente manera a cada tratamiento. Por ejemplo, algunos tratamientos de quimioterapia pueden provocar náuseas, vómitos o disminución del apetito. Es útil que los pacientes anoten sus síntomas para comunicarse con precisión con sus médicos. Por lo tanto, los médicos pueden recomendar medicamentos para aliviar los síntomas y enfrentar mejor el próximo tratamiento. Incluso durante la supervivencia, los pacientes con cáncer son supervisados de por vida y los médicos también pueden prescribirles medicamentos para aliviar el dolor como efecto secundario de los tratamientos. Si bien el objetivo principal es curar el cáncer o prolongar considerablemente la vida, el mantenimiento de la mejor calidad de vida de los pacientes también 
es un objetivo importante. El cuidado paliativo es un tratamiento para aliviar, en lugar de curar, los síntomas causados por el cáncer. Los cuidados paliativos facilitan la transición al final de la vida.

Las observaciones anteriores nos motivaron a diseñar una aplicación móvil para registrar síntomas de pacientes con cáncer, el objetivo es doble: 1) aumentar la conciencia personal del paciente y la responsabilidad personal sobre su enfermedad y 2) proporcionar una herramienta para registrar los síntomas. En el resto del documento, el término "signo vital" se referirá a los signos "que muestran el estado de salud de alguien, como la temperatura corporal, la frecuencia respiratoria y el latido cardíaco". Además, el término "síntoma" se referirá a "cualquier sensación de enfermedad o cambio físico o mental causado por una enfermedad en particular" (Diccionario de Cambridge).

En los últimos años, la Inteligencia Artificial ha incorporado la Interacción Humano Computadora, desde que se desea evitar el desarrollo de soluciones a los problemas de forma aislada; por lo tanto, se ha involucrado la participación de humanos interesados (usuarios) que utilizarán dichas soluciones y que son los más afectados por las mismas. La participación del usuario en el diseño, desarrollo y evaluación de sistemas de inteligencia es fundamental para garantizar que los sistemas basados en inteligencia artificial sean prácticos. En 11, se presentó un enfoque de clasificación supervisada de reportes clínicos, utilizando el algoritmo de Máquinas de Soporte Vectorial, para apoyar el diagnóstico de cuatro tipos de cáncer: estómago, pulmonar, cáncer de pecho y cáncer de piel. Por ello, en este trabajo se presenta la aplicación MoCa que permite el registro de síntomas de pacientes con cáncer.

\section{Trabajo relacionado}

Hay varios trabajos de investigación que están estrechamente relacionados con aumentar la conciencia personal de un paciente y la responsabilidad personal su enfermedad. Wellness Monitor (WM) 4] proporciona a los pacientes un monitoreo continuo de sus signos vitales. Los pacientes tienen sensores en varias partes de su cuerpo. Estos sensores pasan los datos a un accesorio inteligente. Los datos recopilados se envían para su análisis a un dispositivo móvil y luego se envían a una repositorio de datos central. Este repositorio permite a los médicos acceder a los datos de los pacientes. WM realiza la representación de información basada en roles, lo que significa que el paciente solo visualiza sus signos vitales en un informe basado en gráficos, mientras que el médico tiene acceso a todo el repositorio de datos. WM permite a los pacientes con cáncer participar activamente en el monitoreo de su estado de salud. Aunque WM mejora la salud personal, toma signos vitales pero no registra síntomas.

MyMobileDoc [10] es una herramienta móvil diseñada para permitir que los pacientes se involucren activamente en el manejo de sus enfermedades crónicas como la diabetes y la hipertensión. Los pacientes envían sus datos manualmente (presión arterial, niveles de glucosa en la sangre, intensidad del dolor, etc.), desde una aplicación móvil o un navegador de Internet hacia un Centro de 
Datos Médico. Los datos recibidos pueden ser evaluados automáticamente o por personal médico. Con base en estos datos, se envía inmediatamente al paciente una retroalimentación. MyMobileDoc es una aplicación centrada en el paciente que le brinda la posibilidad de aumentar su participación y su conciencia de la salud. Aunque esta aplicación permite registrar la intensidad del dolor, no está claro si se puede especificar el lugar donde ocurre un dolor físico.

MyJourney Compass 5] explora el potencial de las herramientas de gestión de la salud para mejorar la calidad de vida de los pacientes con cáncer. En particular, realizaron un estudio sobre pacientes con cáncer de mama y propusieron información relacionada con la salud facilitando documentos, un conjunto de aplicaciones de salud existentes, enlaces de información de salud y aplicaciones de entretenimiento para ser utilizadas en tabletas. My Journey Compass explora los patrones de uso de los pacientes con cáncer a lo largo de sus jornadas y descubrieron que los pacientes usaban la tableta como ayuda en la organización y como memoria al capturar notas y registrar citas en aplicaciones existentes. Sin embargo, en MyJourney Compass no hay un modelo conceptual sobre los síntomas.

En e-ESAS [3] los pacientes con cáncer de mama son los usuarios principales. Enfocaron su solución en dos conceptos: motivación y automatización. Para aumentar la motivación de los pacientes, desarrollaron dos videos para reducir las barreras existentes que enfrentan los pacientes para continuar el tratamiento. Para obtener datos del paciente automáticamente, desarrollaron un sistema de monitoreo de síntomas basado en dispositivos móviles utilizando el Sistema de evaluación de síntomas de Edmonton (ESAS). Esta herramienta permite a los pacientes registrar sus síntomas utilizando un cuestionario ESAS. Además, los pacientes registran el dolor mínimo, máximo y promedio de las últimas 24 horas. Los médicos pueden ver, para cada paciente, una gráfica longitudinal de un síntoma en un período de tiempo seleccionado. Además, pueden administrar las recetas de los pacientes y crear una generación de alertas basada en condiciones predefinidas sobre el dolor del paciente. Aunque e-ESAS mejora la salud personal y registra síntomas, solo es posible registrar el dolor en una escala de diez, desde "Sin dolor" hasta "El peor dolor posible", pero no es posible registrar dónde se encuentra un dolor físico.

BodyDiagrams [6] es una aplicación en línea para registrar síntomas físicos a través de dibujos y texto. Los pacientes pueden codificar visualmente los atributos de los síntomas, como la gravedad, la frecuencia y la secuencia temporal. BodyDiagrams ofrece un conjunto de patrones de dibujo para indicar una ubicación general o precisa de los síntomas, un campo de texto para describir el síntoma y flechas para asociar las anotaciones del texto a las marcas dibujadas. Además, los pacientes pueden pedir consejo a los médicos. BodyDiagrams mejoró la comunicación entre pacientes y médicos porque los pacientes tuvieron mayor confianza en sus descripciones de BodyDiagrams que en las descripciones de solo texto. Sin embargo, BodyDiagrams solo registra síntomas físicos en modo en línea y no en modo fuera de línea.

Además, existen algunas aplicaciones móviles para el cuidado de la salud. 
ARVmobile v1.0 [8] es una aplicación móvil, de monitoreo de la salud para medir la presión arterial de manera ambulatoria, mejorar la detección temprana y la intervención de la hipertensión; y detectar la presión arterial anormal potencial y la frecuencia cardíaca. Dicha aplicación incluyó sensores para detectar la presión arterial y las señales del ritmo cardíaco y un teléfono inteligente como el receptor para recopilar los datos digitales transmitidos y procesarlos para proporcionar información personalizada, de manera inmediata, al usuario. Los resultados preliminares demuestran que la aplicación ARVmobile v1.0 podría usarse para realizar un perfil detallado de los usuarios. Si bien la aplicación ARVmobile v.1.0 puede ser útil para la atención médica, no permite registrar síntomas.

La salud se ha convertido en un reto para el desarrollo de aplicaciones móviles. En [1] se desarrolló una aplicación móvil para apoyar el aprendizaje al comparar los efectos de dos tipos de aprendizaje diferentes basados en los logros, relacionados con la salud, conseguidos por la actividad física de los estudiantes. La aplicación pudo encontrar los factores clave del diseño de aprendizaje asistido y los logros de los estudiantes para los maestros y los educadores. 9 presenta un accesorio inteligente para el monitoreo de electrocardiogramas (ECG) que integra un sensor inalámbrico de diseño propio para la adquisición de señales de ECG. El sistema podría ser útil para detectar anomalías cardíacas en el entorno familiar y contribuir a la prevención, el diagnóstico oportuno y el tratamiento eficaz de las enfermedades cardiovasculares. [1] y [9] son dos sistemas muy útiles para el monitoreo de la salud y la prevención de enfermedades; sin embargo, no le dan al paciente la posibilidad de expresar síntomas que son de gran utilidad para un diagnóstico temprano.

En este documento, se propone el diseño de una aplicación móvil para registrar síntomas. Aunque esta aplicación puede ser utilizada por cualquier persona/paciente, nuestro usuario principal es un paciente con cáncer. Consideramos a los teléfonos inteligentes como dispositivos específicos debido a su ubicuidad.

\section{Metodología}

Se siguió una metodología colaborativa para el desarrollo de software [2] basada en una versión ágil del Proceso Unificado de Rational [7. Este método se centra en la recopilación de requerimientos en dos fases: inicio y elaboración. En este método, el equipo del proyecto estuvo compuesto por tres diseñadores de interfaz de usuario y tres analistas de sistemas que trabajaron juntos para comprender los requerimientos. En el proceso participaron dos pacientes con cáncer, tres pacientes sin cáncer y un familiar de un paciente con cáncer. Además, se consultó a cinco médicos sobre la pertinencia del proyecto.

El equipo del proyecto llevó a cabo: 1) reuniones, 2) talleres y 3) trabajos individuales después de cada taller tanto en la fase inicial como en la fase de elaboración (ver Fig. 1). Las reuniones sirvieron para capturar y validar los requerimientos de los usuarios. El equipo del proyecto, los pacientes con cáncer y los familiares de los pacientes se reunían al final de la semana. Los talleres 
permitieron al equipo explorar las soluciones potenciales utilizando artefactos como: diagrama de casos de uso, prototipos en papel y electrónicos, escenarios de casos de uso, documentos de visión y de especificación complementaria. Los trabajos individuales permitieron proponer al equipo una solución mediante la construcción de un artefacto. Este método de colaboración siguió un diseño iterativo (consulte las iteraciones I1 y I2 y E1 a E5 de la Fig. 1) y las iteraciones duraron una semana hábil.

\subsection{Captura de los requerimientos del usuario}

Con el fin de capturar los requerimientos de los usuarios, les realizamos entrevistas. Presentamos un extracto de entrevista que se realizó a dos pacientes (Entrevistado P1 y P2):

Entrevistador: ¿El médico considera los síntomas que está sufriendo en el tratamiento del cáncer?

Entrevistado P1: Sí, definitivamente. Cuando estoy en la consulta, el médico me pregunta mis síntomas, luego él decide sobre la dosis de los medicamentos o sobre un cambio de medicamento.

Entrevistado P2: Sí, creo que aunque los síntomas no son decisivos para el tratamiento, el médico los toma en cuenta.

Entrevistador: ¿Le preocupa no recordar sus síntomas durante una consulta? Entrevistado P1: Sí, principalmente al inicio de mi tratamiento, porque no tenía la costumbre de registrarlos.

Entrevistado P2: Sí, creo que un registro de síntomas es esencial. Desde que tengo cáncer, he aprendido a registrar no solo el síntoma, sino también la fecha, la hora, la recurrencia y la intensidad. El médico siempre me presunta estos datos.

Entrevistador: ¿Utiliza una herramienta para controlar los síntomas?

Entrevistado P1: Sólo papel y lápiz. A veces mi esposo registra mis síntomas. Entrevistado P2: Utilizo una agenda digital en mi teléfono inteligente. Escribir mis síntomas en el teléfono es práctico; aunque verlos no es fácil. Solo tengo mis síntomas por fecha pero no puedo consultar por tipo de síntoma, por ejemplo.

Entrevistador: ¿Qué características debería tener una aplicación móvil para ayudarlo en la gestión de los síntomas?

Entrevistado P1: Una aplicación móvil debe permitirme registrar, recuperar y eliminar los síntomas, pero de una manera sencilla.

Entrevistado P2: Una aplicación móvil debería ayudarme a registrar, modificar, recuperar y eliminar los síntomas. Con una aplicación móvil, podría encontrar rápidamente un síntoma por nombre o por un período de tiempo.

Después de entrevistar a dos pacientes con cáncer acerca de su experiencia durante la cita con el médico, expresaron estar preocupados por no poder comunicar todos sus síntomas o por no comunicarlos de manera precisa. Los pacientes recibieron tratamientos para el cáncer en México, en dos hospitales públicos diferentes, donde el número de pacientes que asisten es alto y el tiempo asignado a cada cita es de aproximadamente diez minutos. Le preguntamos a cinco médicos sobre la necesidad de una herramienta móvil para registrar los síntomas de los 
pacientes con cáncer. Al principio, uno de ellos no estaba de acuerdo porque pensaba que la aplicación intentaría reemplazar la comunicación con él. Les explicamos que nuestro objetivo era mejorar la comunicación y apoyar su toma de decisiones, no sustituirla, todos estuvieron de acuerdo con la aplicación.

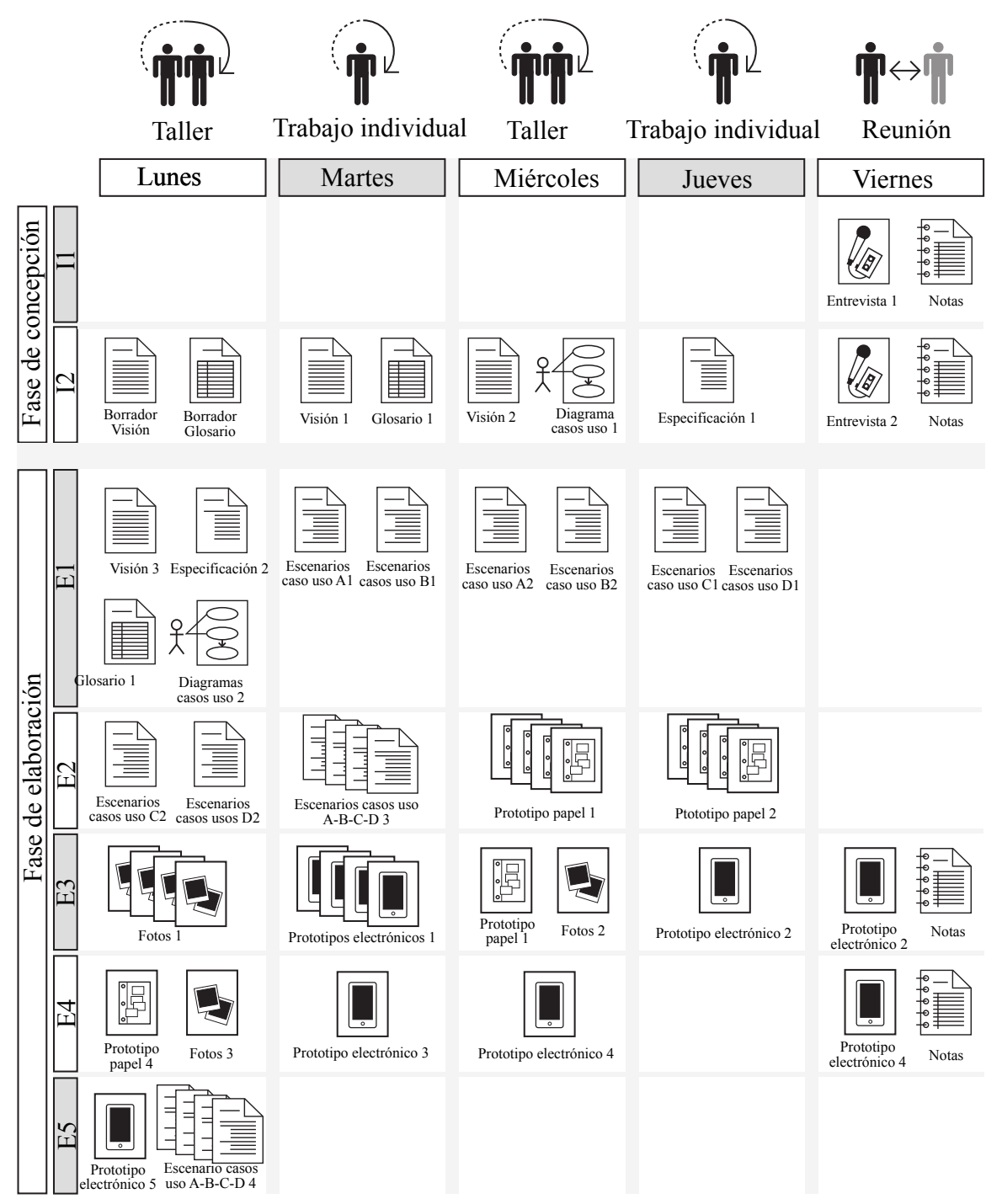

Fig. 1. Sistema MoCa: fases, iteraciones, y artefactos. 


\subsection{Requerimientos MoCa}

Después de entrevistar a los usuarios, los miembros del equipo levantaron los requerimientos de la aplicación MoCa. El objetivo principal de MoCa es ayudar a mejorar la calidad de vida de los pacientes con cáncer, pero este objetivo es doble: 1) aumentar la conciencia personal de un paciente y la responsabilidad personal sobre su enfermedad y 2) proporcionar una herramienta para registrar los síntomas. Si un paciente puede comunicar sus síntomas a sus médicos de manera más precisa, los médicos pueden recetarles medicamentos para aliviar su dolor y así mejorar su calidad de vida. Para ello, los requerimientos de MoCa son:

I. Mejorar la comunicación entre pacientes y médicos. Para mejorar la comunicación, queremos que un paciente pueda registrar y gestionar sus síntomas. Los atributos de los síntomas son: nombre, nivel de intensidad del dolor, descripción, fecha de inicio, fecha de finalización y periodicidad.

II. Mejorar la usabilidad.. Los pacientes con cáncer enfrentan muchas dificultades, tales como: reorganización familiar, permiso de trabajo, etc. MoCa debe proporcionar a los pacientes un fácil aprendizaje de la aplicación y que los pacientes necesiten solo unos segundos para registrar un síntoma. Para facilitar el uso de MoCa, consideramos aprovechar los gestos de teléfonos inteligentes.

III. Permitir el uso en cualquier momento y en cualquier lugar. Para permitir esto, consideramos los teléfonos inteligentes como el dispositivo de destino para soportar esta aplicación.

IV. Permitir registrar síntomas en el modo fuera de línea. Dado que en algunas áreas del hospital y/o en algunas áreas y/o en algunos momentos no hay conexión a Internet, MoCa debe poder registrar los síntomas en el modo fuera de línea.

El requerimiento III se cumplirá mediante la programación de MoCa en las dos plataformas de computación mas importantes: iOS y Android. El requerimiento IV se cumplirá mediante el uso de una base de datos integrada para el almacenamiento local, y al proporcionar una copia de seguridad y restauración de los síntomas del usuario en un servidor remoto. Los requerimientos I y II se integrarán en las funcionalidades de MoCa.

\subsection{Funcionalidades MoCa}

En el transcurso del diseño iterativo, se analizaron las siguientes funcionalidades para MoCa:

1. Registrar un síntoma. Un paciente puede registrar un síntoma de dos maneras:

- Utilizando el teclado. En el momento en que el paciente escribe un síntoma, el sistema los filtra y presenta los síntomas relacionados con las letras tecleadas. 
- Usando un cuerpo humano. El paciente puede seleccionar un área de la figura de un cuerpo humano y el sistema presenta los síntomas asociados con esa área del cuerpo.

Cuando el paciente selecciona un nombre de síntoma e introduce su intensidad (opcionalmente, una breve nota), el sistema considera la fecha y hora actuales para el síntoma. Esta funcionalidad mejorará la usabilidad, por lo tanto, cubrirá el requerimiento II] al proporcionar diferentes modos de entrada.

2. Ver síntomas. El paciente puede ver una lista de los síntomas registrados más recientemente. Esta funcionalidad mejorará la usabilidad y la comunicación entre el paciente y sus médicos, cumpliendo con los requerimientos I y II.

3. Copia de seguridad o recuperación de síntomas. Esta función permite guardar o recuperar los síntomas del paciente a/desde un servidor remoto. Mantener una copia en otro sitio es útil para fines de copia de respaldo.

4. Modificar un síntoma. Cuando el paciente registra un síntoma, no sabe cuándo podría terminar ni su periodicidad. Por lo tanto, el paciente puede actualizar cualquier atributo de un síntoma, excepto su nombre. Esta función le permite al paciente completar un registro de síntomas.

5. Eliminar un síntoma. Esta función permite al paciente eliminar un síntoma. Esta función no se consideró al inicio del proyecto, pero se agregó porque el paciente pudo haberlo registrado por error.

\subsection{Prototipo en papel de MoCa}

Para comprender mejor cómo administrar los síntomas para mejorar la comunicación y la usabilidad, se implementaron prototipos en papel de un teléfono inteligente y creamos una secuencia de fotos de su uso (ver Fig. 2). El primer boceto (Principal) muestra la figura de un cuerpo humano a través del cual es posible registrar los síntomas. Después, un paciente puede seleccionar una región del cuerpo humano y el sistema mostrará el conjunto de síntomas que pueden asociarse con la región del cuerpo seleccionada;el paciente puede seleccionar un síntoma (Captura de síntomas por región anatómica). Los últimos bocetos (Resumen de síntomas) le permiten buscar un síntoma escribiendo su nombre y, más tarde, modificarlo.

\subsection{Aplicación MoCa}

En esta sección, presentamos la aplicación MoCa y cómo cumple con los requerimientos presentados. La Fig. 3 muestra la interfaz que está presente al abrir MoCa por primera vez. Esta interfaz muestra un mensaje de bienvenida donde el usuario escribe su nombre, coloca su género y selecciona su fecha de nacimiento (ver Fig. 3a)). Una vez que se presiona el botón "Listo", el sistema presenta la figura de un cuerpo humano (masculino o femenino) según el género indicado en la interfaz de bienvenida. En la parte inferior de la Fig. 3b), hay cuatro íconos que describen las funcionalidades principales de $\mathrm{MoCa}$ (de izquierda a derecha): 
Beatriz A. González-Beltrán, Lizbeth Gallardo-López, Roberto García-Madrid, et al.

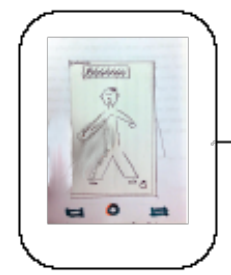

Principal

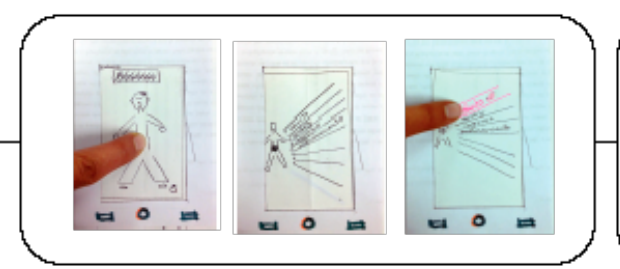

Captura de síntomas por región anatómica

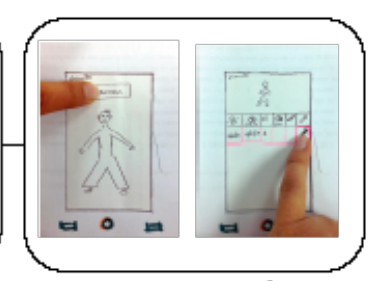

Resumen de síntomas

Fig. 2. Secuencia de fotos de prototipos en papel de la aplicación MoCa.

registrar un síntoma utilizando el teclado, ver la parte posterior del cuerpo humano, hacer copia de seguridad o restaurar los síntomas del usuario, y ver la lista de síntomas. Estos íconos permiten alcanzar con un solo toque la mayoría de las funcionalidades de $\mathrm{MoCa}$, mejorando así la usabilidad (requerimiento III). Además, la Fig. 33) muestra la lista de los síntomas (Resumen). Cuando el usuario selecciona el ícono "lápiz", puede editar el síntoma. Por ejemplo, cuando el usuario selecciona el ícono "lápiz" debajo de la etiqueta "Fecha de finalización", el sistema permite al usuario registrar la fecha en que desapareció el síntoma. La interfaz "Ver síntomas" permite a los usuarios ver fácilmente la lista de síntomas, mejorando así la facilidad de uso (requerimiento II) y permitiendo recordar los síntomas para comunicarse con el médico (requerimiento I).

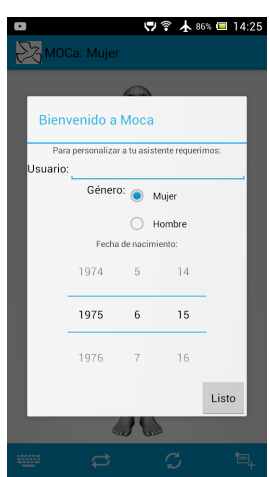

a)

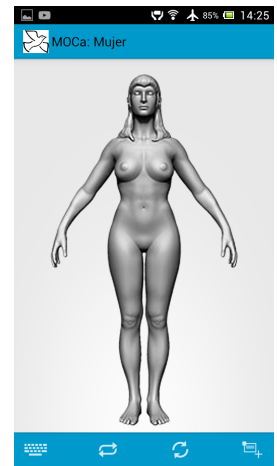

b)

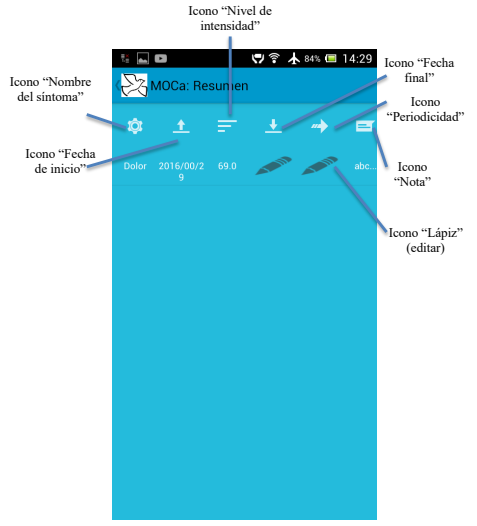

c)

Fig. 3. Interfaces MoCa: a) Bienvenido a MoCa, b) Cuerpo humano y c) Ver síntomas.

El cuerpo humano permite al usuario registrar un síntoma seleccionando una región anatómica (ver Fig. 4). Por ejemplo, cuando el usuario toca la cabeza del cuerpo humano (ver Fig. 4a), el sistema presenta una lista de síntomas asociados 
con esta región (vea Fig. 4 b). Cuando el usuario selecciona el dolor de cabeza, el sistema presenta una ventana de "nivel de intensidad del dolor". El usuario puede usar el control deslizante para seleccionar entre 1 y 10 (ver Fig. 4p)) y puede describir el síntoma (por ejemplo, intenso) y puede aceptar o cancelar el síntoma. Los gestos táctiles de apoyo permiten a los usuarios seleccionar regiones para registrar fácilmente un síntoma, mejorando así la facilidad de uso (requerimiento II).

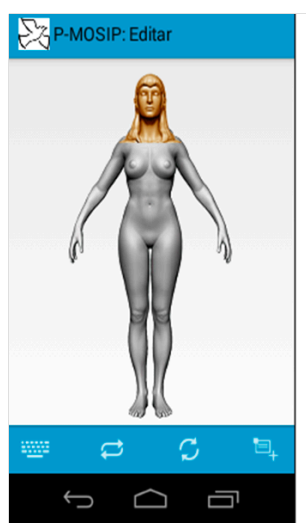

a)

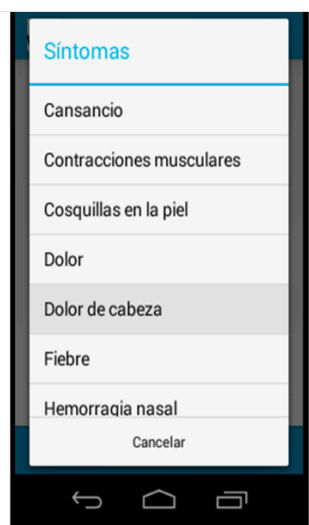

b)

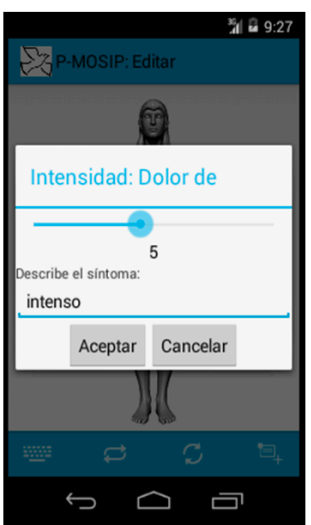

c)

Fig. 4. Registrar un síntoma utilizando MoCa.

\section{Resultados y discusión}

MoCa ha sido desarrollado para Android Jelly Bean 4.3. Las funcionalidades de copia de seguridad y restauración se implementaron como servicios RESTful en un servidor remoto. MoCa es un cliente RESTful que solicita servicios de copia de respaldo y restauración.

Se realizaron pruebas cualitativas y se les pidió a los pacientes que habían evaluado la funcionalidad de MoCa que enviaran un comentario de forma libre. Se les pidieron comentarios sobre las fortalezas y debilidades de cada interfaz. Todos los participantes respondieron positivamente, les gustó la aplicación. Una de las ventajas citadas de un paciente con cáncer incluye la facilidad para registrar un síntoma: "en mi opinión, el cuerpo humano me permite registrar un síntoma facilmente, simplemente tocando la figura de un cuerpo humano, elegir un síntoma y elegir el nivel de intensidad del dolor. Cuando vuelvo a casa después del tratamiento contra el cáncer, me siento agotado y necesito descansar, pero puedo registrar mis síntomas de una manera fácil, así que lo hago". A otro 
paciente de cáncer le gustó la lista de síntomas y dijo: 'La lista de síntomas me permite comunicar mis dolores a los médicos al consultar el resumen en una ventana en lugar de tener que buscar en mi agenda". Sin embargo, este usuario dijo: "Me confunden algunos íconos, en particular la ventana de visualización de síntomas, tuve que preguntar el significado de algunos de ellos".

Para discutir el trabajo realizado, se relacionan las funcionalidades y los requerimientos de la siguiente manera:

- El paciente puede, de una manera fácil, registrar un síntoma seleccionando una región de un cuerpo humano o escribiendo el nombre del síntoma; por lo que se mejora la usabilidad de MoCa. Se proporcionan dos modos de registro porque un paciente puede tener confianza con una forma humana para explicar su síntoma o puede tener más confianza al escribir el nombre del síntoma. La navegación tiene la flexibilidad suficiente para permitir que los pacientes naveguen en ambos modos.

- El soporte para registrar y modificar un síntoma se realiza con solo tres pasos y se solicita una cantidad mínima de información al registrar un síntoma; por lo que se mejora la usabilidad de MoCa. Además, esta tarea de uso frecuente está disponible de inmediato. Al abrir MoCa, el paciente puede registrar un síntoma seleccionando un área del cuerpo humano. Esta tarea mejora la usabilidad de MoCa.

- El paciente puede ver sus síntomas con solo un paso. El paciente solo toca el ícono "Ver síntomas" y puede visualizar sus últimos síntomas registrados con todas sus características (nombre del síntoma, nivel de intensidad, notas, fecha de inicio, fecha de finalización y periodicidad) en una sola fila. Esta vista mejora la usabilidad de MoCa y permite al paciente describir sus síntomas de una manera precisa; por lo que la comunicación entre el paciente y sus médicos es más precisa.

- El paciente puede modificar, desde la interfaz "Ver síntomas", un atributo de un síntoma solo presionando el ícono "lápiz" debajo del atributo a modificar. Los pacientes pueden cambiar fácilmente un registro de síntomas. Esta característica mejora la usabilidad de MoCa. Sin embargo, los usuarios se confunden con algunos íconos dentro de esta interfaz. Antes de la implementación de MoCa, se debe realizar un trabajo adicional para proponer íconos más informativos en esta interfaz.

- El paciente puede eliminar, desde la interfaz "Ver síntomas", un síntoma con tan solo deslizar hacia la izquierda el renglón del síntoma y enviarlo a la "Papelera". El paciente tiene la oportunidad de confirmar la acción de eliminación. De esta manera, los pacientes pueden eliminar fácilmente un síntoma, pero al mismo tiempo, el sistema proporciona retroalimentación. Esta característica mejora la usabilidad de MoCa.

- El paciente puede respaldar y restaurar sus síntomas a/desde un servidor remoto. Esta función mejora la confidencialidad de la aplicación, porque en caso de falla de un teléfono inteligente, sus registros de síntomas están respaldados. Sin embargo, los usuarios prefieren hacer una copia de seguridad y restaurar desde la nube, en lugar desde un servidor en particular. 


\section{Conclusiones y trabajo a futuro}

Este documento ha presentado los resultados del diseño de MoCa, una aplicación móvil para registrar síntomas de pacientes con cáncer mediante la realización de entrevistas con los usuarios. El registro de síntomas se llevó a cabo utilizando una figura corporal o un teclado.

Nuestra contribución es proporcionar a los pacientes con cáncer una aplicación móvil para registrar sus síntomas de una manera fácil. Este problema es relevante porque los pacientes deben aprender a ser conscientes de sus signos y síntomas pero hay que tener en cuenta que pueden estar en tratamiento, están agotados y debe ser fácil de usar. Los pacientes deben comunicar sus síntomas a sus médicos para mantener una mejor calidad de vida.

La aplicación móvil obtenida (MoCa) puede ayudar a los médicos a tomar decisiones con pacientes con cáncer, como mejorar la monitorización, la continuidad del tratamiento y la calidad de vida del paciente.

Nuestro plan es modificar los íconos que no fueron relacionados fácilmente con sus funciones y agregar la función de copia de seguridad y restauración desde la nube. Una vez que se hayan hecho estas modificaciones, planeamos realizar una prueba de usabilidad mas grande con pacientes en un hospital oncológico.

Además, como trabajo futuro, nuestra aplicación MoCa considera registrar el tratamiento realizado, los eventos adversos en los pacientes o las alergias a los medicamentos. Sería muy útil si el paciente pudiera registrar estos aspectos utilizando la voz como interfaz de comunicación. Por último, se contempla generar modelos de clasificación supervisada aplicada a los síntomas de los pacientes.

Agradecimientos. Agradecemos la participación de los pacientes y médicos que apoyaron durante el diseño de MoCa. Los autores agradecen ampliamente a la Universidad Autónoma Metropolitana por el apoyo al proyecto no. SI001-14.

\section{Referencias}

1. Cheng, C.H., Chen, C.H.: Developing a Mobile APP-Supported Learning System for Evaluating Health-Related Physical Fitness Achievements of Students. Mobile Information Systems 2018, 15 (2018), https://doi.org/10.1155/2018/8960968

2. Gallardo-Lopez, L., Gonzalez-Beltran, B.A., Garcia-Madrid, R., Ferruzca, M., Zafra-Ballinas, I.A., Reyes-Ortiz, J.A.: Collaborative working: Understanding mobile applications requirements. In: 2015 International Conference on Computational Science and Computational Intelligence (CSCI). pp. 139-142 (Dec 2015)

3. Haque, M., Kawsar, F., Adibuzzaman, M., Ahamed, S., Love, R., Dowla, R., Roe, D., Hossain, S., Selim, R.: Findings of e-esas: A mobile based symptom monitoring system for breast cancer patients in rural bangladesh. In: Proceedings of the SIGCHI Conference on Human Factors in Computing Systems. pp. 899-908. CHI '12, ACM, New York, NY, USA (2012), http://doi.acm.org/10.1145/2207676.2208532

4. Islam, R., Ahamed, S.I., Talukder, N., Obermiller, I.: HCI and Usability for Medicine and Health Care: Third Symposium of the Workgroup Human-Computer 
Beatriz A. González-Beltrán, Lizbeth Gallardo-López, Roberto García-Madrid, et al.

Interaction and Usability Engineering of the Austrian Computer Society, USAB 2007 Graz, Austria, November, 22, 2007. Proceedings, chap. Usability of Mobile Computing Technologies to Assist Cancer Patients, pp. 227-240. Springer Berlin Heidelberg, Berlin, Heidelberg (2007)

5. Jacobs, M.L., Clawson, J., Mynatt, E.D.: My journey compass: A preliminary investigation of a mobile tool for cancer patients. In: Proceedings of the SIGCHI Conference on Human Factors in Computing Systems. pp. 663-672. CHI '14, ACM, New York, NY, USA (2014), http://doi.acm.org/10.1145/2556288.2557194

6. Jang, A., MacLean, D.L., Heer, J.: Bodydiagrams: Improving communication of pain symptoms through drawing. In: Proceedings of the SIGCHI Conference on Human Factors in Computing Systems. pp. 1153-1162. CHI '14, ACM, New York, NY, USA (2014), http://doi.acm.org/10.1145/2556288.2557223

7. Larman, C.: Applying UML and Patterns: An Introduction to Object-Oriented Analysis and Design and Iterative Development. Prentice Hall, Upper Saddle River, N.J, third edn. (Oct 2004)

8. Mena, L.J., Felix, V.G., Ostos, R., Gonzalez, J.A., Cervantes, A., Ochoa, A., Ruiz, C., Ramos, R., Maestre, G.E.: Mobile Personal Health System for Ambulatory Blood Pressure Monitoring. Computational and Mathematical Methods in Medicine 2013, 13 (2013), http://dx.doi.org/10.1155/2013/598196

9. Mena, L.J., Félix, V.G., Ochoa, A., Ostos, R., González, E., Aspuru, J., Velarde, P., Maestre, G.E.: Mobile Personal Health Monitoring for Automated Classification of Electrocardiogram Signals in Elderly. Computational and Mathematical Methods in Medicine 2018, 9 (2018), https://doi.org/10.1155/2018/9128054

10. Nischelwitzer, A., Pintoffl, K., Loss, C., Holzinger, A.: HCI and Usability for Medicine and Health Care: Third Symposium of the Workgroup Human-Computer Interaction and Usability Engineering of the Austrian Computer Society, USAB 2007 Graz, Austria, November, 22, 2007. Proceedings, chap. Design and Development of a Mobile Medical Application for the Management of Chronic Diseases: Methods of Improved Data Input for Older People, pp. 119-132. Springer Berlin Heidelberg, Berlin, Heidelberg (2007)

11. Reyes-Ortiz, J.A., González-Beltrán, B.A., Vidal, M.T.: Clasificación de reportes clínicos para apoyar el diagnóstico del cáncer. Pistas Educativas 39(128), 1347-1361 (febrero 2018)

12. World Health Organization: Global Status Report on noncommunicable diseases 2014. WHO Press, Switzerland (2014) 